\title{
Accurate Localization with Ultra-Wideband: Tessellated Spatial Models and Collaboration
}

\author{
Amanda Prorok and Alcherio Martinoli
}

\begin{abstract}
Ultra-wideband (UWB) localization is a recent technology that promises to outperform many indoor localization methods currently available. Despite its desirable traits, such as precision and high material penetrability, the resolution of non-line-of-sight (NLOS) signals remains a very hard problem and has a significant impact on the localization accuracy. In this work, we address the peculiarities of UWB error behavior by building models that capture the spatiality as well as the multimodal nature of the error statistics. Our framework utilizes tessellated maps that associate multimodal probabilistic error models to localities in space. In addition to our UWB localization strategy (which provides absolute position estimates), we investigate the effects of collaboration in the form of relative positioning. We test our approach experimentally on a group of ten mobile robots equipped with UWB emitters and extension modules providing inter-robot relative range and bearing measurements.
\end{abstract}

\section{Introduction}

Due to its large frequency spectrum, UWB is able to penetrate through objects in NLOS scenarios, and thus alleviates the LOS constraint imposed by other sensor types relying on media such as infrared, ultrasound, visible light or narrow-band radio. This advantage ultimately enables localization over large ranges and in dynamic environments [5], which makes UWB an attractive candidate for indoor applications such as asset management, inventory tracking and assembly control, for a variety of different industries. Nevertheless, NLOS scenarios may cause biases in the signal propagation times, which leads to significant localization errors. In order to guarantee reliable and accurate performance, these biases need to be addressed by an effective localization strategy.

Amanda Prorok and Alcherio Martinoli

Distributed Intelligent Systems and Algorithms Laboratory, School of Architecture, Civil and Environmental Engineering, Ecole Polytéchnique Fédérale de Lausanne (EPFL), Switzerland e-mail: \{amanda.prorok, alcherio.martinoli\}@epfl.ch 
In this paper, we consider the problem of absolute localization of a team of mobile robots for unknown initial conditions. We design an algorithm that works in conjunction with a tessellated spatial error model built a priori. Our solution targets miniaturized platforms equipped with low-power sensing modalities, and we ultimately envision its portability onto much smaller devices such as embedded/portable tags. Our localization strategy uses time-difference-of-arrival (TDOA) measurements from two or more UWB base station pairs and on-board deadreckoning information. Lastly, as it is commonly known that multi-robot collaboration is able to compensate for deficiencies in the data owned by a single robot [1, 7], we extend our approach to include relative (inter-robot) range and bearing observations.

Recently, UWB has received some attention within the robotics community. The studies in [2] and [4] develop probabilistic models for biased UWB range measurements which are combined with on-board odometry data. Yet, both papers model NLOS biases within augmented-state particle filters that do not take LOS/NLOS path conditions and bias probability distributions into account explicitly, and that depend on the motion of the mobile target. Furthermore, given the novelty of UWB positioning systems in the robotics community, to the best of our knowledge, no significant studies have been performed on the fusion of UWB with on-board exteroceptive sensors, in the case of single-robot systems, nor any on-board relative positioning sensors, in the case of multi-robot systems. Lastly, this work is amongst the first to model UWB TDOA errors as a function of space.

\section{Technical Approach}

Our proposed method is a culmination of our ongoing research efforts in the domain of indoor localization, combining two main elements: an UWB system capable of absolute positioning [10, 12], and a collaborative multi-robot system capable of relative observations [8, 11].

\subsection{UWB Localization}

UWB is a radio technology which is characterized by its very large bandwidth compared to conventional narrowband systems, and in particular features high positioning accuracy (due to a time resolution in the order of nanoseconds), and high material penetrability (due to a bandwidth typically larger than $0.5 \mathrm{GHz}$ ). Despite these desirable traits, the resolution of multipath signals remains a hard problem-the complexity of implementing state-of-the-art direct signal path detection algorithms is exacerbated by the necessity of maintaining very high sampling rates (in the order of several GHz). Thus, our approach addresses the occurrence of both LOS and NLOS signal paths by proposing a probabilistic measurement model that captures this multimodal error behavior. But because NLOS biases are originally introduced by the clutter in a given environment, the perceived error behavior is actually a spa- 
tial phenomenon. Thus, even if a non-spatial error model is able to represent the error distribution in mixed LOS/NLOS signal path environments, a spatial model will very likely produce better results. Yet, given the sharp (discontinuous) transitions from LOS to potentially harsh NLOS localities in space, developing a viable spatial UWB error model is a hard problem. The goal of this work is indeed to develop such a spatial error model. Given the reasons elaborated above, we resort to a mapping technique that allows us to tessellate space into areas, and where each area is associated with a unique error model. Indeed, we exploit the ability of our error model to capture and adapt to all types of UWB propagation, and spatially customize the parametrization of this model using the underlying tessellation (to ultimately produce a map, which can be fine-tuned according to user requirements).

When using UWB for localization, the measured quantity is time-of-flight. However, in practice, time-of-arrival (TOA) systems are rarely implemented due to the complexity induced by the required synchronization of a mobile node with the base stations. Instead, it is a common choice to implement time-difference-of-arrival (TDOA) systems which are significantly more practical, since only the synchronization among base stations is required. This, in turn, enables a significant miniaturization of the emitter boards (to an order of a few centimeters in size), as well as a reduction of the consumption power (to an order of $\mathrm{mW}$ ).

\subsubsection{Framework}

We consider a pair of UWB base stations $\left\langle\mathscr{B}_{u}, \mathscr{B}_{v}\right\rangle$, both fixed and well-localized in an absolute coordinate system, and a robot $\mathscr{R}_{n}$ at position $\mathbf{x}_{n}$, equipped with an UWB emitter tag. At any given time, the robot $\mathscr{R}_{n}$ may receive a measured TDOA value $\hat{\tau}_{u v, n}$ from any pair of base stations $\left\langle\mathscr{B}_{u}, \mathscr{B}_{v}\right\rangle$. We denote by $T_{n, t}=$ $\left\{\left\langle\hat{\tau}_{u v, n, t},\left\langle\mathscr{B}_{u}, \mathscr{B}_{v}\right\rangle\right\rangle \mid \exists\left\langle\mathscr{B}_{u}, \mathscr{B}_{v}\right\rangle \in \mathbb{B}\right\}$ the set of TDOA measurements received by a robot $\mathscr{R}_{n}$ at a given time $t$. The TDOA measurement error $\Delta \tau_{u v, n}$ for robot $\mathscr{R}_{n}$ and base station pair $\left\langle\mathscr{B}_{u}, \mathscr{B}_{v}\right\rangle$ is defined as the difference between the nominal (errorfree) TDOA value at the actual robot position and the measured TDOA value

$$
\Delta \tau_{u v, n}\left(\hat{\tau}_{u v, n}, \mathbf{x}_{n}\right)=\hat{\tau}_{u v, n}-\tau_{u v, n}\left(\mathbf{x}_{n}\right),
$$

where $\tau_{u v, n}\left(\mathbf{x}_{n}\right)=r_{u}\left(\mathbf{x}_{n}\right)-r_{v}\left(\mathbf{x}_{n}\right)$, and $r_{u}\left(\mathbf{x}_{n}\right)$ is the range between base station $\mathscr{B}_{u}$ and $\mathbf{x}_{n}$. In order to model the UWB error behavior, we take account of spatiality by defining a set $\mathscr{M}_{u v}$ of $N_{A}$ areas $\mathscr{M}_{u v}=\left\{\left\langle A_{a}, \boldsymbol{\theta}_{u v, a}\right\rangle \mid a=1, \ldots, N_{A}\right\}$ where $\boldsymbol{\theta}_{u v, a}$ is a parameter vector, and $A_{a} \subset \mathbb{R}^{2}$. Note that the areas are disjoint $\bigcap_{n} A_{a}=\emptyset$ and $\bigcup_{n} A_{a}$ covers the whole space. In other words, each area $A_{a}$ is associated with a parameter vector $\boldsymbol{\theta}_{u v, a}$, as illustrated in Figure 1(b)] We refer to $\mathscr{M}_{u v}$ as the map for base station pair $\left\langle\mathscr{B}_{u}, \mathscr{B}_{v}\right\rangle$, and denote the set of all maps as $\mathscr{M}=\left\{\mathscr{M}_{u v} \mid \exists\left\langle\mathscr{B}_{u}, \mathscr{B}_{v}\right\rangle \in \mathbb{B}\right\}$, where $\mathbb{B}$ is the set of all base station pairs. Furthermore, we define a function $m_{u v}: \mathbb{R}^{2} \mapsto \Theta$ that maps to any position in two-dimensional space a parameter vector in the finite set $\Theta: m_{u v}\left(\mathbf{x}_{n}\right)=\boldsymbol{\theta}_{u v, a}$ such that $\exists\left\langle A_{a}, \boldsymbol{\theta}_{u v, a}\right\rangle \in \mathscr{M}_{u v}$ with $\mathbf{x}_{n} \in A_{a}$. Finally, we model the error $\Delta \tau_{u v, n}\left(\hat{\tau}_{u v, n}, \mathbf{x}_{n}\right)$ for a given base station pair $\left\langle\mathscr{B}_{u}, \mathscr{B}_{v}\right\rangle$ with a probability density function $p$ that covers an area $A_{a}$ (such that $\mathbf{x}_{n} \in A_{a}$ ) and that depends on 


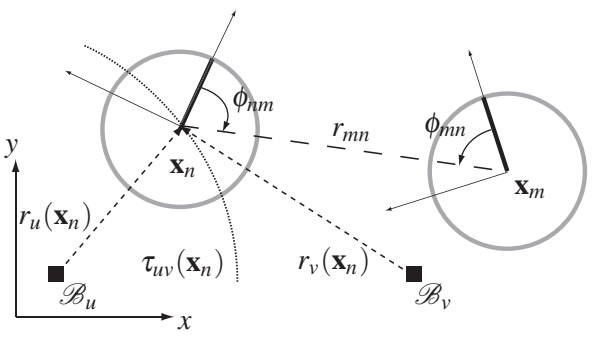

(a)

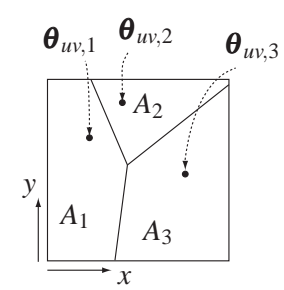

(b)

Fig. 1 (a) System of two robots at positions $\mathbf{x}_{n}, \mathbf{x}_{m}$ and two well-localized UWB base stations $\mathscr{B}_{u}$ and $\mathscr{B}_{v}$. The figure shows the true ranges $r_{u}\left(\mathbf{x}_{n}\right)$ and $r_{v}\left(\mathbf{x}_{n}\right)$ of robot $\mathscr{R}_{n}$ to the respective base stations, as well as a segment of the hyperbola resulting from the range-difference measurement $\tau_{u v, n}$. The figure also depicts the relative robot range $r_{m n}=r_{n m}$, and the relative bearing values $\phi_{m n}$ and $\phi_{n m}$. (b) Distinct UWB error models $p\left(\Delta \tau_{u v, n} ; \boldsymbol{\theta}_{u v, a}\right)$ are mapped to individual areas $A_{a}$.

the parameter vector $\boldsymbol{\theta}_{u v, a}$. We define our error model as

$$
p\left(\Delta \tau_{u v, n} ; \boldsymbol{\theta}_{u v, a}\right) \triangleq p\left(\Delta \tau_{u v, n}\left(\hat{\tau}_{u v, n}, \mathbf{x}_{n}\right) ; m_{u v}\left(\mathbf{x}_{n}\right)\right) .
$$

Practically, we solve the problem of localizing with UWB measurements in two steps. First, we collect a data set covering the working environment to build the maps in $\mathscr{M}$. Second, during the actual exercise, we use $\mathscr{M}$ to look up UWB error models in function of the current estimated positions, and then use these models within a localization filter to evaluate incoming UWB observations.

\subsubsection{UWB Error Model}

Our baseline error model [14] for the range 1 between a base station $\mathscr{B}_{u}$ and a target node (robot) at position $\mathbf{x}_{n}$ is

$$
\hat{r}_{u}=r_{u}\left(\mathbf{x}_{\mathbf{n}}\right)+\varepsilon+Y b_{u}
$$

where $r_{u}\left(\mathbf{x}_{n}\right)$ represents the true distance, $b_{u}$ is a non-negative distance bias introduced by a NLOS signal propagation, and $\varepsilon \sim p_{\mathscr{N}}\left(0, \sigma_{N}^{2}\right)$ is a zero-mean Gaussian measurement noise with variance $\sigma_{\mathscr{N}}^{2}$, common to all base stations. The bias $b_{u}$ is modeled as a log-normal random variable $b_{u} \sim p_{\ln \mathscr{N}}\left(\mu_{u}, \sigma_{u}\right)$, supported on the semi-infinite interval $(0, \infty)$, and which is associated uniquely to a base station $\mathscr{B}_{u}$. The random variable $Y$ qualifies the occurrence of a NLOS signal path and follows a Bernoulli distribution. Explicitly, it takes the value 1 with probability $\left(1-\mathrm{P}_{L_{u}}\right)$ and the value 0 with probability $\mathrm{P}_{L_{u}}$, where $\mathrm{P}_{L_{u}}$ is the probability of measuring a LOS path, and correspondingly, $\left(1-\mathrm{P}_{L_{u}}\right)$ is the probability of measuring a NLOS path.

${ }^{1}$ The terms TOA and TDOA are used interchangeably with the terms range and range difference, respectively, as they differ only by a constant factor (propagation speed). 
For a range error defined as

$$
\Delta r_{u}\left(\hat{r}_{u}, \mathbf{x}\right)=\hat{r}_{u}-r_{u}(\mathbf{x})
$$

the TOA measurement model $p_{u}$ describes the likelihood of $\Delta r_{u}$ occurring when a robot measures a certain range distance $\hat{r}_{u}$ from a base station $\mathscr{B}_{u}$ at an actual position $\mathbf{x}$ with a nominal (actual) range $r_{u}$ (we refer the reader to [12] for a detailed derivation of $p_{u}$ ).

Then, in order to model TDOA instead of TOA observations, we define the difference range value (i.e. TDOA) between two base stations $\mathscr{B}_{u}$ and $\mathscr{B}_{v}$ to a target node $\mathscr{R}_{n}$ and model the TDOA error $\Delta \tau_{u v}$ as previously shown in Equation 1 . Given this formalism, we can describe the probability density of a given TDOA measurement error $\Delta \tau_{u v}$ as the probability density of the subtraction of two random variables drawn from the probability densities $p_{u}$ and $p_{v}$, describing the TOA error models of the two respective base stations. The resulting probability density is

$$
p_{u v}\left(\Delta \tau_{u v}\right) \triangleq\left(p_{u} * p_{v}^{-}\right)\left(\Delta \tau_{u v}\right)
$$

which is a convolution of the probability density of the range error $\Delta r_{u}$ and the mirrored probability density of $\Delta r_{v}$ (i.e., $p_{v}^{-}\left(\Delta r_{v}\right)=p_{v}\left(-\Delta r_{v}\right)$ ).

Although numerical implementations for the TDOA measurement model of Equation 5] are easily found, they imply nested integrals which may incur a substantial computational overhead when deploying the model on a real embedded platform for real-time operation. Also, the model itself is analytically non-tractable, which causes difficulties when deriving viable estimators. For these reasons, we use a closed-form approximation [12] to simplify the TDOA measurement model of Equation 5 to a sum of four terms:

$$
\begin{aligned}
p\left(\Delta \tau_{u v} ; \boldsymbol{\theta}_{u v, a}\right)=( & \mathrm{P}_{L_{u}} \mathrm{P}_{L_{v}} p_{\sqrt{2} \mathscr{N}}+\mathrm{P}_{L_{u}}\left(1-\mathrm{P}_{L_{v}}\right) p_{\ln \mathscr{N}, v}^{-}+ \\
& \left.\mathrm{P}_{L_{v}}\left(1-\mathrm{P}_{L_{u}}\right) p_{\ln \mathscr{N}, u}+\left(1-\mathrm{P}_{L_{u}}\right)\left(1-\mathrm{P}_{L_{v}}\right) p_{\tilde{N}}\right)\left(\Delta \tau_{u v}\right)
\end{aligned}
$$

Here, $p_{\sqrt{2} \mathscr{N}}=p_{\mathscr{N}} * p_{\mathscr{N}}^{-}$, and $p_{\mathscr{N}}$ approximates the convolution $p_{\ln \mathscr{N}} * p_{\ln \mathscr{N}}^{-}$with a Gaussian that matches its moments. Hence, the parameter vector $\boldsymbol{\theta}_{u v, a}$ is

$$
\boldsymbol{\theta}_{u v, a}=\left[\mu_{u}, \sigma_{u}, \mu_{v}, \sigma_{v}, \mathrm{P}_{L_{u}}, \mathrm{P}_{L_{v}}\right]^{\top}
$$

and $\mu_{u}, \mu_{v} \in \mathbb{R}, \sigma_{u}, \sigma_{v} \in \mathbb{R}^{+}$, and $\mathrm{P}_{L_{u}}, \mathrm{P}_{L_{v}} \in[0,1]$. Finally, we note that, given some ground truth TDOA measurement errors, the parameters of the above equation can be determined via an efficient Expectation Maximization algorithm (batch mode as well as online) that maximizes their likelihood [12]. Figure 2 provides an intuition of the multimodal nature of TDOA error data, and illustrates how our error model proposes to capture this. In particular, Figure 2(b) illustrates how each of the four terms of Eq. 6 represents one of the four possible modes of operation for a base station pair: LOS-LOS, NLOS-LOS, LOS-NLOS, and NLOS-NLOS. 


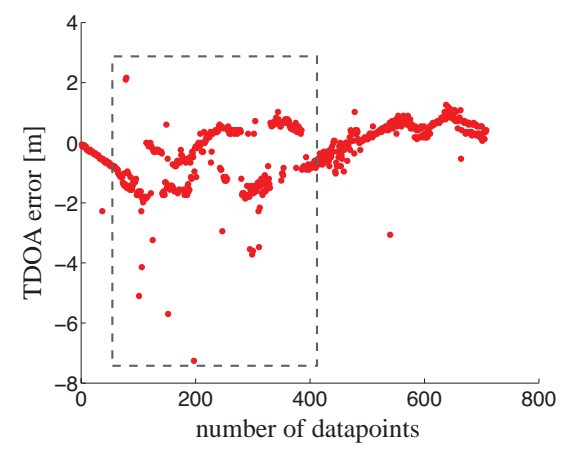

(a)

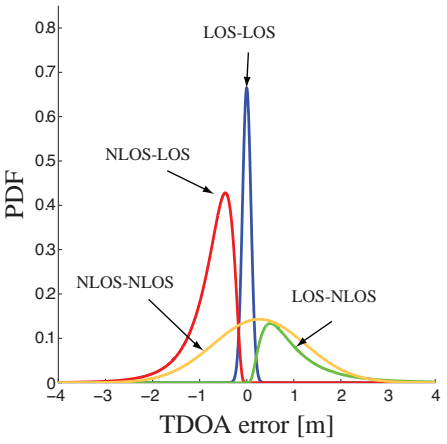

(b)

Fig. 2 (a) Example of the multimodal nature of TDOA error data, in particular those points contained within the dashed box. This data was collected by a robot moving in a straight line through an indoor environment with obstacles. (b) We consider a base station pair $\left\langle\mathscr{B}_{1}, \mathscr{B}_{2}\right\rangle$. The plot illustrates the four modes which form the complete multimodal probability density function shown in Eq. 6 The model parameters are set to: $\mu_{1}=-0.43, \mu_{2}=-0.2, \sigma_{1}=0.6, \sigma_{2}=0.7, \mathrm{P}_{L_{1}}=0.3$, $\mathrm{P}_{L_{2}}=0.5$.

\subsection{Collaborative Localization and UWB}

There is abundant literature discussing various strategies toward solving the multirobot localization problem. Our approach distinguishes itself particularly by respecting the following design goals: low cost, full decentralization and scalability, and asynchrony of relative observations [8, 12]. Given its efficiency in solving the localization problem for unknown initial conditions and its ability to accommodate arbitrary probability density functions, our method of choice is the particle filter (otherwise known as Monte Carlo Localization (MCL)). Our collaboration strategy exploits associated, inter-robot relative range and bearing observations, which are evaluated by a dedicated detection model and fused with dead-reckoning information (e.g., odometry) to form position estimates.

We subsequently introduce an augmented system by considering both relative positioning data as well as UWB, schematized in Figure 1(a) Essentially, we propose a baseline algorithm which fuses UWB TDOA measurements and relative positioning measurements with dead-reckoning information. Ultimately, the goal is to experimentally test this algorithm and provide an insight into if and how multi-robot strategies can contribute to improving the accuracy of UWB.

\subsubsection{Framework}

Our multi-robot system is composed of $N_{\mathscr{R}}$ robots $\mathscr{R}_{1}, \mathscr{R}_{2}, \ldots, \mathscr{R}_{N_{\mathscr{R}}}$, each running an individual localization filter. The belief of a robot's pose is formulated as 


$$
\operatorname{Bel}\left(\mathbf{x}_{n, t}\right) \sim\left\{\left\langle\mathbf{x}_{n, t}^{[i]}, w_{n, t}^{[i]}\right\rangle \mid i=1, \ldots, M\right\}=X_{n, t}
$$

where $M$ is the number of particles, $\mathbf{x}_{n, t}^{[i]}$ is a sample of the random variable $\mathbf{x}_{n, t}=\left[x_{n, t}, y_{n, t}, \psi_{n, t}\right]^{\top}$ (where $x_{n, t}$ and $y_{n, t}$ are Euclidian coordinates and $\psi_{n, t}$ is the orientation), and $w_{n, t}^{[i]}$ is its weight. The symbol $X_{n, t}$ refers to the set of particles $\left\langle\mathbf{x}_{n, t}^{[i]}, w_{n, t}^{[i]}\right\rangle$ at time $t$ belonging to robot $\mathscr{R}_{n}$. At any given time $t$, a robot $\mathscr{R}_{m}$ may make a range measurement $\hat{r}_{m n, t}$ and a bearing measurement $\hat{\phi}_{m n, t}$ of robot $\mathscr{R}_{n}$. Thus, with the knowledge of the range and bearing noise values, we define a robot detection model $q$ which describes the probability that robot $\mathscr{R}_{m}$ detects $\mathscr{R}_{n}$ at position $\mathbf{x}_{n, t}$, given the detection data $d_{m n, t}=\left\langle\hat{r}_{m n, t}, \hat{\phi}_{m n, t}, X_{m, t}\right\rangle$, as

$$
q\left(\mathbf{x}_{n, t} \mid d_{m n, t}\right) \triangleq q\left(\mathbf{x}_{n, t} \mid \hat{r}_{m n, t}, \hat{\phi}_{m n, t}, X_{m, t}\right) .
$$

\subsubsection{Sensor Fusion Algorithm}

The routine is shown here in Algorithm 1, Line 3 shows the application of the motion model, where $u_{n, t}$ represents dead-reckoning information. Line 4 shows the application of the measurement model where $T_{n, t}$ represents the TDOA data. Line5 shows the application of the robot detection model, where $D_{n, t}=\left\{d_{m n, t} \mid \mathscr{R}_{m} \in \mathscr{N}_{n, t}\right\}$ is the set of all communication messages received by robot $\mathscr{R}_{n}$. In other words, the detected robot will apply the detection model using data received from the robots that made the detection. A more detailed description of our robot detection model can be found in [11]. In addition to using the robot detection model for updating the belief representation Bel $\left(\mathbf{x}_{n, t}\right)$, our approach relies on a reciprocal sampling method [8], shown in line 13 .

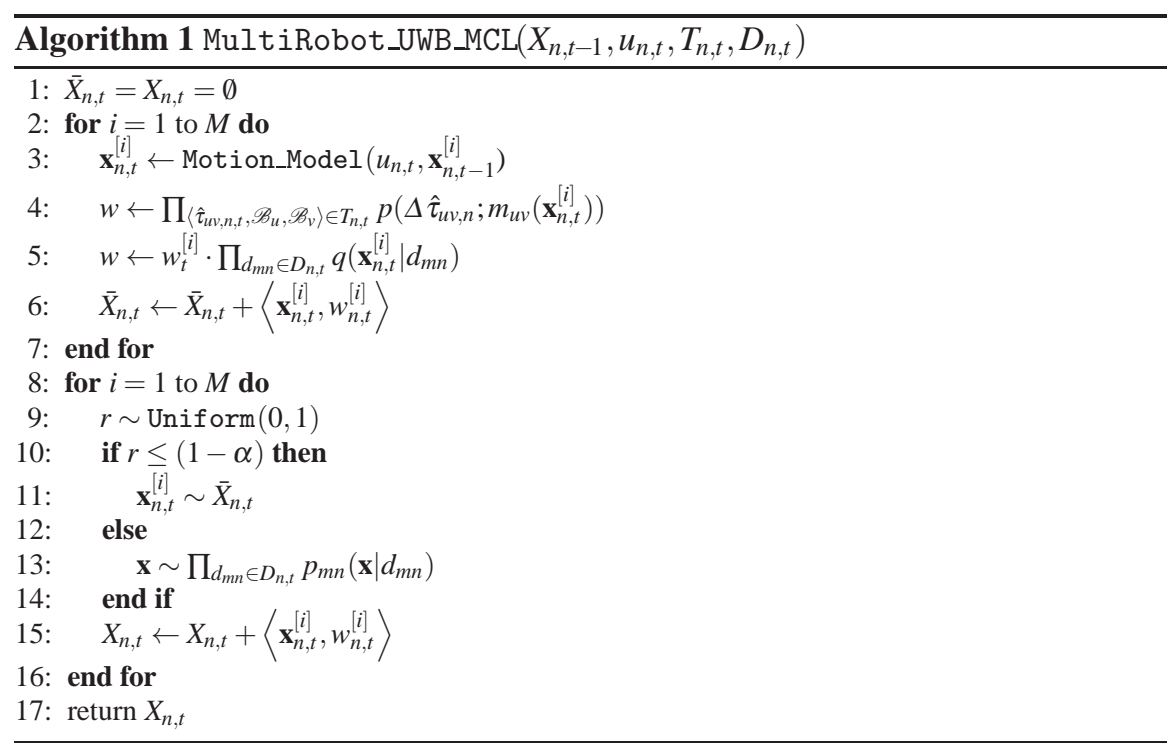




\section{Experiments}

The following section details our experimental setup. We then elaborate the practical implementation of our framework, as introduced in Section 2.1.1

\subsection{Setup}

Our experimental setup (Figure 3(a) consists of three main elements $(i)$ a group of ten mobile robots, (ii) an UWB positioning system composed of four base stations, and (iii) two overhead cameras with overlapping views of a $5 \times 3 \mathrm{~m}^{2}$ arena.

To perform experiments, we use ten Khepera III robots that drive randomly in the arena at a speed of one robot size per second. The Khepera III is a differential drive robot of $12 \mathrm{~cm}$ diameter produced by K-Team corporation 2 , see Figure 4 . We use the robot with a KoreBot II extension board providing a standard embedded Linux operating system on an Intel XSCALE PXA-270 processor running at $624 \mathrm{MHz}$. Communication is enabled through an IEEE 802.11b wireless card which is installed in a built-in CompactFlash slot. The robot uses wheel encoders to provide odometry

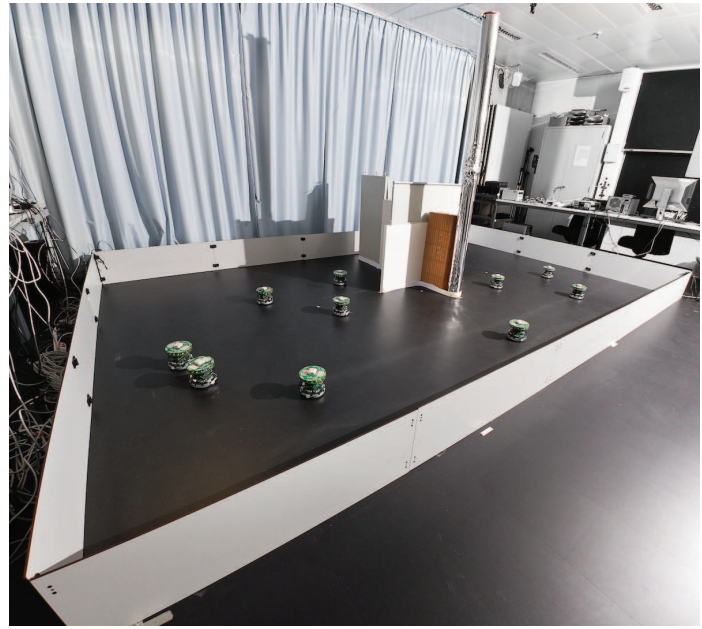

(a)

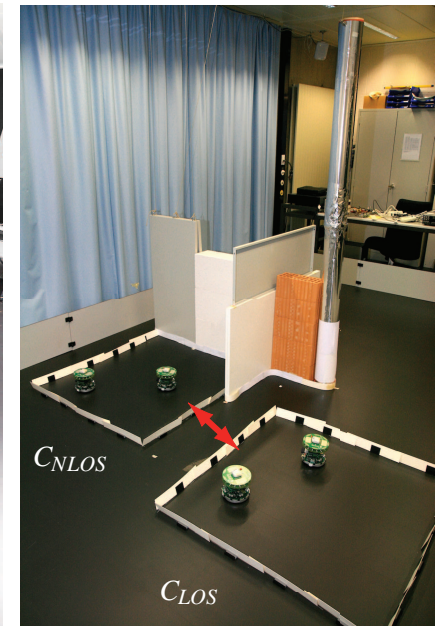

(b)

Fig. 3 (a) The $5 \times 3 \mathrm{~m}^{2}$ experimental arena contains an obstacle composed of various elements made of brick, plaster, metal, wood, and a 3 meter high tube covered in aluminum. Four UWB base stations are mounted on the ceiling in the corners of the lab room. Two overhead cameras provide ground truth positioning in the experimental area. (b) In an additional setup, we test the benefits of collaboration by confining the directions of intercellular robot detections in between a strongly occluded cell (and thus, predominantly NLOS cell, $C_{N L O S}$ ) and a predominantly LOS cell $\left(C_{L O S}\right)$.

\footnotetext{
${ }^{2}$ http://www.k-team.com/
} 
Fig. 4 The Khepera III robot is equipped with a range and bearing extension module which utilizes sixteen infrared LEDs. On top of this board, we mounted an active marker (LED) tracking module, which simultaneously carries the UWB emitter tag.

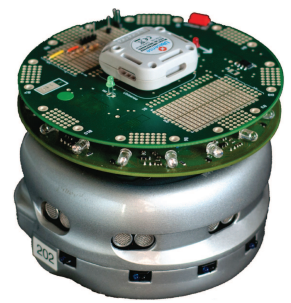

readings at $5 \mathrm{~Hz}$. It also uses a relative range and bearing module [13], which is set to provide the measurements used by the detection model at a frequency of $1 \mathrm{~Hz}$. The modules' noise characteristics were empirically determined in our actual setup $\left(\sigma_{r}=0.15 \cdot r_{m n}\right.$, and $\left.\sigma_{\phi}=0.15 \mathrm{rad}\right)$. We set its maximum detection range to be $1 \mathrm{~m}$. Lastly, the robot is also equipped with an LED-based active marker module for tracking that also carries the UWB emitter tag, which emits positioning pulses at a frequency of $10 \mathrm{~Hz}$. The UWB localization system employed in this work is commercially available from Ubisense 3 , Series 7000 (sensors and compact tags). It is installed on the ceiling, in the corners of our $40 \mathrm{~m}^{2}$ laboratory. The overhead camera system runs on a central processor which also enacts the synchronization of available ground truth positioning data with all incoming raw sensor data (from the UWB system as well as from the robots). In order to compute the ground truth robot positions, the camera system utilizes the open source tracking software SwisTrack [6]. The average error of the resulting ground truth is roughly $1 \mathrm{~cm}[9])$.

In order to create a NLOS setting that occludes direct paths between the UWB emitters carried by the robots and the four base stations, we install a cross-shaped obstacle in our arena (see Figure 3(a) . The obstacle is $1.5 \mathrm{~m}$ long, $1 \mathrm{~m}$ high and $20 \mathrm{~cm}$ thick, and is composed of several modules made of various materials (brick, plaster, metal, wood). At its extremity, in the center of the arena, we attach a $2 \mathrm{~m}$ high tube covered in aluminum. These shapes and materials are chosen in order to realistically emulate the various effects of a typical indoor environment on UWB propagation. Finally, Figure 3(b) shows a secondary setup, which we use to test collaborative strategies more explicitly (as discussed later in Section 4.2).

\subsection{Mapping}

Since we consider three base station pairs $\left(\left\langle\mathscr{B}_{1}, \mathscr{B}_{2}\right\rangle,\left\langle\mathscr{B}_{1}, \mathscr{B}_{3}\right\rangle,\left\langle\mathscr{B}_{1}, \mathscr{B}_{4}\right\rangle\right), \mathscr{M}$ is composed of three maps $\mathscr{M}_{12}, \mathscr{M}_{13}$, and $\mathscr{M}_{14}$. We use the robots to collect a data set comprising over 50'000 TDOA values $\hat{\tau}_{u v}$ for each base station pair $\left\langle\mathscr{B}_{u}, \mathscr{B}_{v}\right\rangle$, and record the associated ground truth positions $\mathbf{x}$, guaranteeing full coverage of our experimental arena. For each data point $\hat{\tau}_{u v}$, we then calculate the ground truth TDOA value $\tau_{u v}(\mathbf{x})$, which, in turn, allows us to compute the TDOA error value $\Delta \tau_{u v}$ and associate it to the position $\mathbf{x}$. Figure 5 visualizes the resulting data set

\footnotetext{
${ }^{3}$ http://www.ubisense.net
} 
of average TDOA error values as a function of space. We observe the higher bias averages of up to $\pm 2 \mathrm{~m}$ in the vicinity of the obstacle (note that TDOA errors are positive as well as negative). Also, we note that although this figure helps us identify strongly biased areas, the plotted average error values do not accurately represent the full multimodal error behavior.

We now proceed with the creation of our set of maps $\mathscr{M}$ to be used by the UWB localization algorithm. In order to build these maps, we discretize our space into $N_{A}=375$ equally sized grid cells of dimension $0.2 \times 0.2 \mathrm{~m}^{2}$, with at least 150 data points per grid cell and per base station pair. For each cell $A_{a}, a \in\left\{1, \ldots, N_{A}\right\}$ defined by our grid map, and for each of the three base station pairs, we now estimate (using Expectation Maximization, as described in Section 2.1.2 the model parameters $\hat{\boldsymbol{\theta}}_{u v, a}$ that define a unique TDOA error model per cell. This concludes the mapping step, and we denote the final set of maps $\mathscr{M}_{M M, 375}$.

As a way of validating our multimodal error model, we calculate an additional set of maps (with the same resolution as above) composed of Gaussian distributions instead of our proposed error model (Eq. 6). Thus, in the same way as above, we associate a mean and variance value to each cell in each of the three maps. The final set of maps comprising (unimodal) Gaussian distributions is denoted $\mathscr{M}_{U M, 375}$. Furthermore, in order to test the effect of spatiality on localization performance, we build two more sets of maps (one with our multimodal error model, and one with Gaussians), composed of one single $5 \times 3 \mathrm{~m}^{2}$ cell. We denote these two sets by $\mathscr{M}_{M M, 1}$ and $\mathscr{M}_{U M, 1}$.

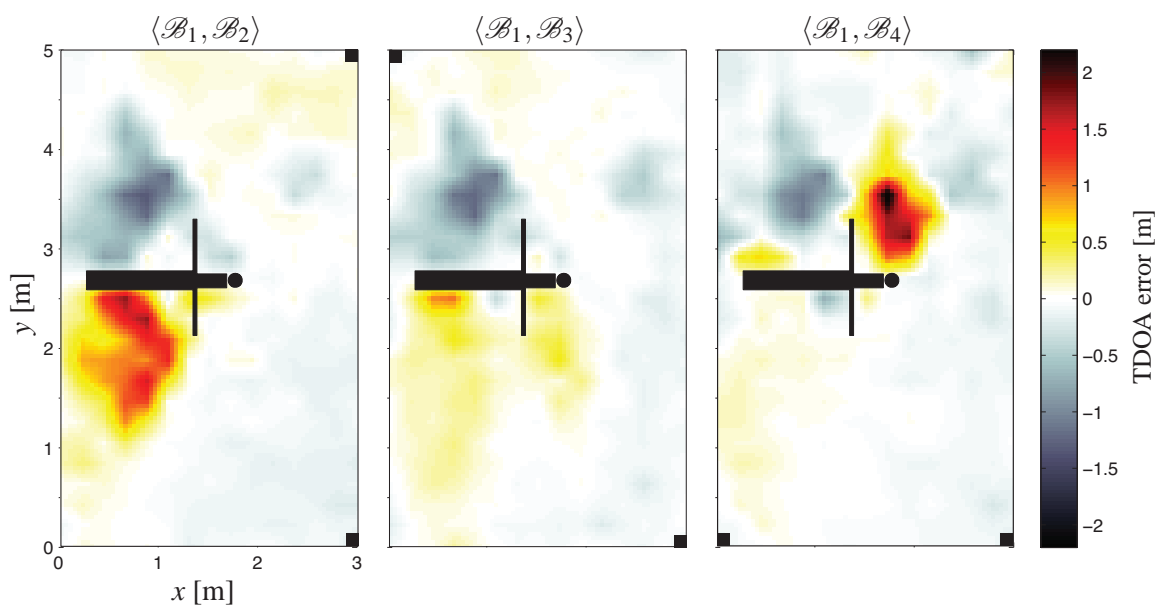

Fig. 5 Overhead view of the experimental arena showing the average TDOA error as a function of space, for three base station pairs (we perform 2D smoothing with a Gaussian kernel of size 1 on a grid map of $150 \times 250$ cells, using over 50 '000 UWB TDOA measurements per base station pair). The cross shape shows the placement of the obstacle and the filled squares in the panel corners schematically indicate the placements of the base stations with respect to the layout of the experimental arena. 
To illustrate the concept of our mapping strategy, Figure 6 shows fits of (i) a Gaussian probability density function, and (ii) the probability density function proposed by our error model in Eq. 6, for 3 difference cell sizes, a) $0.5 \times 0.5 \mathrm{~m}^{2}$, b) $1 \times 1 \mathrm{~m}^{2}$ and c) $2 \times 2 \mathrm{~m}^{2}$. We note that in all cases, the multimodal approach (as proposed by our error model) suggests a better fit to the data. Also, we see that the multimodal nature of the error statistics is preserved across different scales.

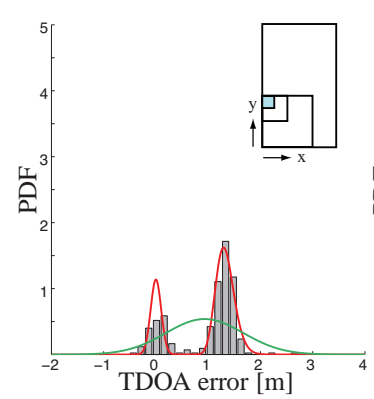

(a)

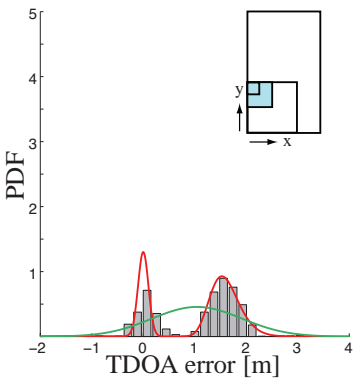

(b)

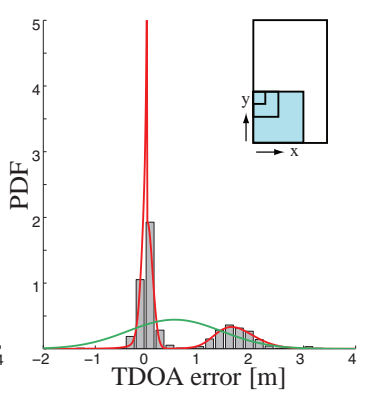

(c)

Fig. 6 Normalized histograms of TDOA error data for base station pair $\left\langle\mathscr{B}_{1}, \mathscr{B}_{2}\right\rangle$. We fit a Gaussian (in green) onto the data, as well as our proposed error model of Eq. 6 (in red). The data is collected over a (a) $0.5 \times 0.5 \mathrm{~m}^{2}$ large square, (b) $1 \times 1 \mathrm{~m}^{2}$ large square, and (c) $2 \times 2 \mathrm{~m}^{2}$ large square. The areas of data collection are indicated by the shaded cells in the schematized arena, in the top right corner of each panel.

\section{Results}

The paragraphs below discuss two distinct experiments.

\subsection{Overall localization error}

Our localization algorithm is evaluated on a data set comprising a 40 minute experiment involving ten robots, initially randomly distributed over the arena. Each robot runs Algorithm 1 with 100 particles, which are initially uniformly distributed in the arena (this problem is otherwise known as global localization). Apart from the modalities described in this paper, the robots use no other sensors to localize. We discuss the localization performance in terms of the positioning error (distance to ground truth position) of the center of mass of the particles in a robot's belief, for all ten robots used in the experiment. We test the four mapping strategies (Gaussian vs. multimodal, and 1 cell vs. 375 cells) with and without collaboration-for the non-collaborative version, we omit the robot detection model (Algorithm 1 line 5).

Figure 7 shows the localization performance as a function of space. We note the irregularity of the error distribution: higher errors tend to be in the vicinity of the obstacle. The plots indicate that our multimodal model $\left(\mathscr{M}_{M M}\right)$ is better than the 


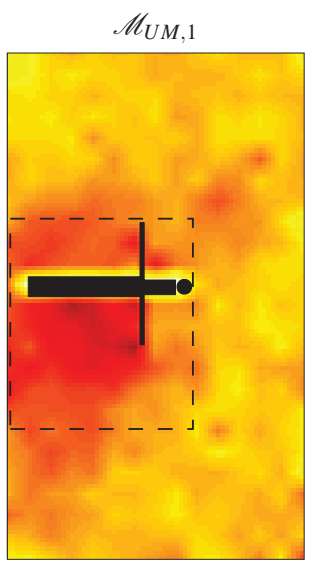

$\mathscr{M}_{U M, 375}$
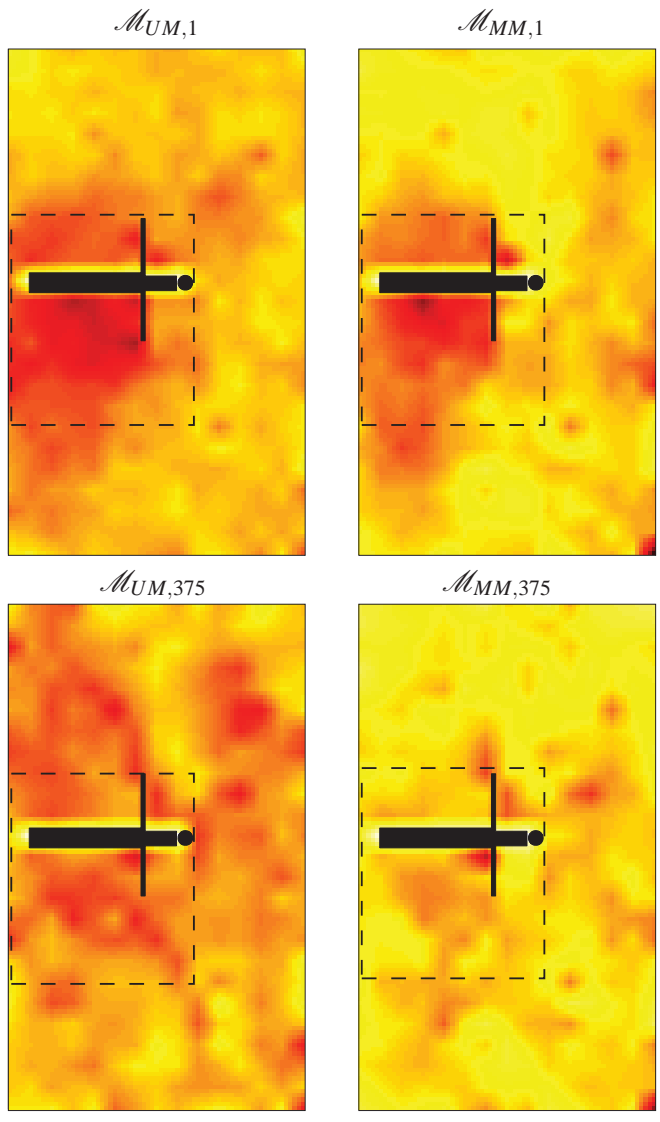

$\mathscr{M}_{M M, 375}$
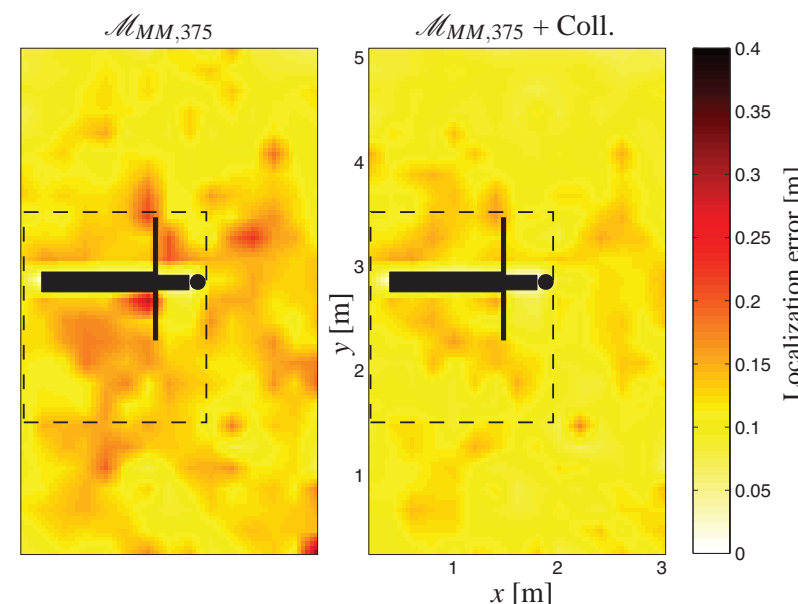

Fig. 7 The graphs show the average localization error as a function of space, over a 40 minute experiment with ten robots. Six different models are tested. On the top row, the robots used the maps composed of one single cell, and on the bottom row, the robots used the maps composed of 375 cells. The first column shows results for the Gaussian maps, the second column for the multimodal error model maps, and the last column uses multimodal maps as well as collaboration. The dashed line delimits the critical area $C_{o b s}$ around the obstacle.

Gaussian $\left(\mathscr{M}_{U M}\right)$, and that a high resolution map (375 cells) is better than a very low resolution map ( 1 cell). Furthermore, the results indicate that collaboration may mitigate errors, in particular in areas prone to high errors due to NLOS: the bottom right panel ( $\mathscr{M}_{M M, 375}+$ Coll. $)$ shows a constant distribution of localization errors in the order of $10 \mathrm{~cm}$.

In order to better understand the performance behavior in critical (NLOS) areas, Figure 8 discusses the localization errors measured inside the area $C_{o b s}$ as marked by a dashed line in Figure 7 Figure 8(a) summarizes the results without collaboration, in the form of boxplots. We can observe that for a fixed granularity, the multimodal model outperforms the Gaussian model. Also, increasing the model granularity im- 
proves localization performance. Figure $8(\mathrm{~b})$ summarizes the results with collaboration, in the form of boxplots. For three of the four cases, the performance improves (with a maximum improvement of $18 \%$ ) with respect to the results of Figure $8(\mathrm{a})$ In the best case $\left(\mathscr{M}_{M M .375}\right)$, we have a median localization error of $7.6 \mathrm{~cm}$. Finally, for comparison, we note that the median of the maximum likelihood trilateration estimates (computed with raw TDOA measurements) amounts to $56 \mathrm{~cm}$.

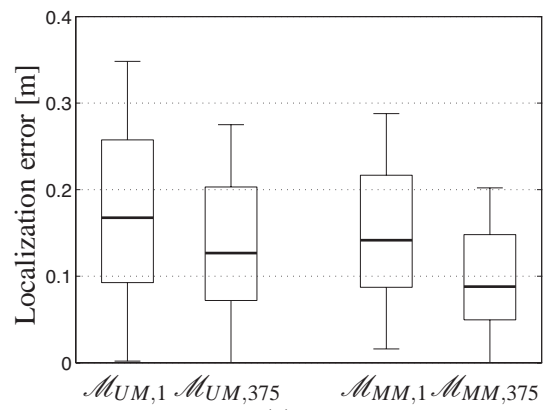

(a)

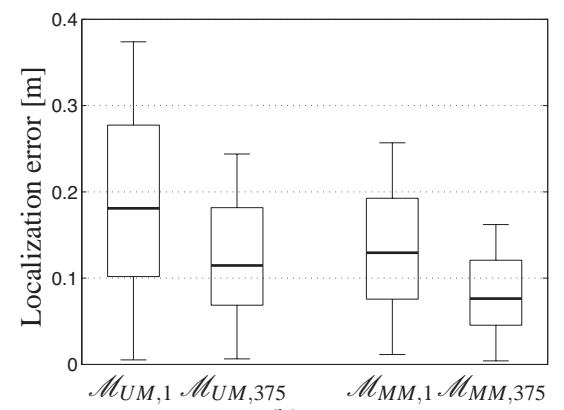

(b)

Fig. 8 Localization error of all ten robots for an experimental run of 40 minutes duration. (a) Without collaboration. (b) With collaboration. The results are shown in the form of boxplots (25th, 50th and 75 th percentile and whiskers containing $85 \%$ of the data). Only errors in $C_{o b s}$ are considered.

\subsection{Evaluation of Collaboration}

The previous section establishes that improved performance can be obtained when collaboration is exploited. Here, we look at a more targeted experiment to better understand how collaboration contributes to this improvement. We use the setup shown in Figure 3(b), with two groups of two robots each. The robots' motion is delimited by the cell boundaries, which simultaneously defines a new set of maps (in this case, we denote our set of maps $\mathscr{M}_{M M, 2}$, using 2 cells of size $1 \mathrm{~m}^{2}$ each). Also, as can be observed when comparing Figure 5 to Figure 3(b), the cell $C_{N L O S}$ is located in a region where significant bias values occur (in particular for base station pair $\left\langle\mathscr{B}_{1}, \mathscr{B}_{2}\right\rangle$ ), whereas cell $C_{L O S}$ is located in a relatively benign region. Importantly, we note that the cell boundaries are low enough to enable intercellular detections via the relative positioning modules. This setting allows us to test the following collaborative configurations (we remind that reader that if robot $\mathscr{R}_{m}$ detects robot $\mathscr{R}_{n}$, it is robot $\mathscr{R}_{n}$ that will execute the sensor fusion using detection data sent by robot $\mathscr{R}_{m}$, see Section 2.2.2): (i) robots in $C_{L O S}$ detect robots in $C_{N L O S}$, (ii) robots in $C_{N L O S}$ detect robots in $C_{L O S}$, and (iii) any detections are allowed. Figure 9(b)] shows the performances of the three variant strategies. Clearly, method $(i)$ produces the best results: LOS environments benefit from a high accuracy (due to an essentially unimodal distribution with a narrow peak, as shown in Figure 9(a)], and thus, it is beneficial to allow robots in $C_{L O S}$ to influence the beliefs of the robots in $C_{N L O S}$. 

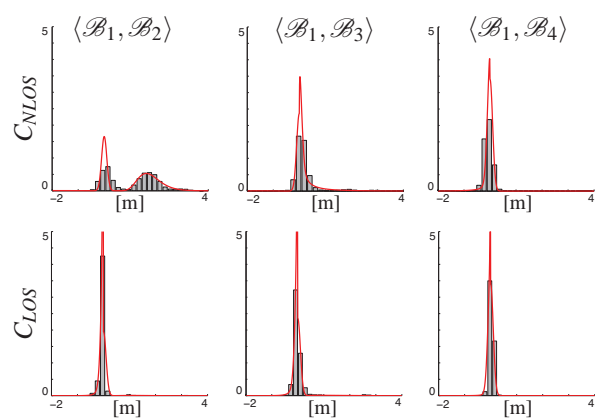

(a)

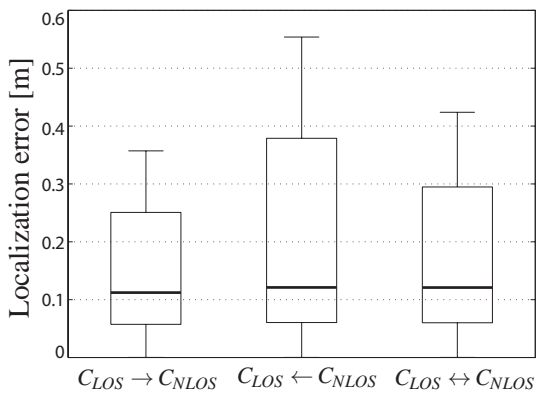

(b)

Fig. 9 (a) Normalized histograms of TDOA error data for all base station pairs (columns) and both cells $C_{L O S}$ and $C_{N L O S}$ (rows). We fit our proposed error model of Eq. 6 onto the data, and use the resulting PDF in the set of maps $\mathscr{M}_{M M, 2}$. (b) Localization error of four robots (as shown in Figure 3(b)], employing $\mathscr{M}_{M M, 2}$ and using three variant collaboration schemes, for an experimental run of 20 minutes duration. The results are shown in the form of boxplots.

\section{Experimental Insights}

Although, in theory, UWB localization has the potential of providing centimeter level accuracy, in practice, sophisticated strategies are necessary to mitigate the effect of NLOS biases. Our experiments showed that the error behavior of time-offlight based UWB measurements is dependent on the configuration of the environment, and thus, can be modeled as a function of space. In conclusion, this work has allowed us to make three main insights: (I) There is a clear benefit in terms of localization accuracy when using our multimodal error model instead of a unimodal Gaussian error model. We have seen that even when using the lowest map granularity, the multimodal model almost matches the performance of the unimodal, Gaussian model with the highest granularity. This conclusion is additionally strengthened by the five-fold performance improvement over the maximum likelihood estimates. (II) High resolution maps (i.e. with small cell sizes) result in higher localization accuracy. However, it is to be assumed that low resolution maps may be equally good, given that the cell separations faithfully separate LOS from NLOS areas, as well as separate differing NLOS cells from each other. (III) A performance increase can be obtained by fusing relative positioning information with UWB data, even when the relative positioning data is potentially noisy. Furthermore, by identifying LOS

and NLOS cells, targeted collaboration strategies can be designed, which promise to even further improve the localization performance.

\section{Acknowledgement}

The work presented in this paper was supported by the National Competence Center in Research on Mobile Information and Communication Systems (NCCR-MICS), 
a center supported by the Swiss National Science Foundation under grant number 51NF40-111400.

\section{References}

[1] D. Fox, W. Burgard, H. Kruppa, and S. Thrun. A Probabilistic Approach to Collaborative Multi-Robot Localization. Autonomous Robots, 8:325-344, 2000.

[2] J. Gonzalez, J. L. Blanco, C. Galindo, A. Ortiz-de Galisteo, J. A. Fernandez-Madrigal, F. A. Moreno, and J. L. Martinez. Mobile robot localization based on Ultra-Wide-Band ranging: A particle filter approach. Robotics and Autonomous Systems, 57(5):496-507, 2009.

[4] D.B. Jourdan, J.J. Jr Deyst, M. Z. Win, and N. Roy. Monte Carlo localization in dense multipath environments using UWB ranging. In IEEE International Conference on UltraWideband (ICU), pages 314-319, 2005.

[5] H. Liu, H. Darabi, P. Banerjee, and J. Liu. Survey of Wireless Indoor Positioning Techniques and Systems. IEEE Transactions on Systems, Man and Cybernetics, 37(6):1067-1080, 2007.

[6] T. Lochmatter, P. Roduit, C. Cianci, N. Correll, J. Jacot, and A. Martinoli. SwisTrack - A Flexible Open Source Tracking Software for Multi-Agent Systems. In Proceedings of the 2008 IEEE/RSJ International Conference on Intelligent Robots and Systems, pages 4004 4010, 2008.

[7] A.I. Mourikis and S. I. Roumeliotis. Performance analysis of multirobot cooperative localization. IEEE Transactions on Robotics, 22(4):666-681, 2006.

[8] A. Prorok and A. Martinoli. A Reciprocal Sampling Algorithm for Lightweight Distributed Multi-Robot Localization. In Proceedings of the 2011 IEEE/RSJ International Conference on Intelligent Robots and Systems (IROS), pages 3241-3247, 2011.

[9] A. Prorok, A Arfire, A. Bahr, J R Farserotu, and A. Martinoli. Indoor navigation research with the Khepera III mobile robot: An experimental baseline with a case-study on ultrawideband positioning. In International Conference on Indoor Positioning and Indoor Navigation (IPIN), 2010. doi: 10.1109/IPIN.2010.5647880.

[10] A. Prorok, P. Tomé, and A. Martinoli. Accommodation of NLOS for Ultra-Wideband TDOA Localization in Single- and Multi-Robot Systems. In International Conference on Indoor Positioning and Indoor Navigation (IPIN), 2011. doi: 10.1109/IPIN.2011.6071927.

[11] A. Prorok, A. Bahr, and A. Martinoli. Low-Cost Collaborative Localization for LargeScale Multi-Robot Systems. In IEEE International Conference on Robotics and Automation (ICRA), pages 4236-4241, 2012.

[12] A. Prorok, L. Gonon, and A. Martinoli. Online Model Estimation of Ultra-Wideband TDOA Measurements for Mobile Robot Localization. In IEEE International Conference on Robotics and Automation (ICRA), pages 807-814, 2012.

[13] J. Pugh, X. Raemy, C. Favre, R. Falconi, and A. Martinoli. A Fast On-Board Relative Positioning Module for Multi-Robot Systems. IEEE Transactions on Mechatronics, 14(2):151$162,2009$.

[14] Z. Sahinoglu, S. Gezici, and I. Guvenc. Ultra-wideband Positioning Systems. Theoretical Limits, Ranging Algorithms, and Protocols. Cambridge University Press, 2008. 